\title{
Métricas rítmicas en tres dialectos Amper-España
}

\author{
Rhythmic metrics in three dialects of Amper-Spain \\ Guillermo Toledo \\ Consejo Nacional de Investigaciones Científicas y Técnicas, Québec (QC), Canadá. \\ Correo electrónico: guillermo.toledo@sympatico.ca
}

Con el fin de refinar la clase rítmica de una lengua de isocronía silábica, el español, e indicar variaciones dialectales, se calculan cuatro métricas rítmicas liberadas de perturbaciones producidas por los cambios en la velocidad de habla: (1) la proporcionalidad de intervalos vocálicos $(\% \mathrm{~V})$, (2) el índice de variabilidad de los intervalos vocálicos en pares, normalizados por la media de esos pares (n PVI-V), (3) la desviación estándar de intervalos consonánticos normalizados por logaritmos de base e (Delta V) y (4) una métrica de control, el índice de variabilidad de los intervalos consonánticos en pares, normalizados por la media de esos pares (n PVI-C). Los resultados separan las lenguas romances (el español) de las lenguas germánicas. Asimismo, indican diferencias dialectales.

Palabras clave: ritmo en lenguas germánicas, ritmo en lenguas romances, clase rítmica, métricas rítmicas, diferencias dialectales en español.

With the purpose of refining the rhythmic class of a syllable-timed language like Spanish and also indicating dialect variations, four normalized metric rhythmic to avoid disturbances caused by changes in speaking rate: (1) the proportionality of vocalic intervals (\% V), (2) the index of variability of vocalic intervals in pairs, normalized by the average of those pairs (n PVI-V), (3) the standard deviation of consonantal intervals normalized by logarithm base e ( $\mathrm{n}$ Delta $\mathrm{V}$ ), and (4) a metric of control, the index variability of consonantal intervals in pairs, normalized by the average of those pairs (n PVI-C) are calculated. The results separate the Romance languages (Spanish) of the Germanic languages. They also indicate dialectal differences.

Key words: rhythm in Germanic languages, rhythm in Romance languages as Spanish; rhythmic class, rhythmic metrics, dialectal differences in Spanish.

\section{INTRODUCCIÓN}

Abercrombie (1967) considera que existen dos tipos de ritmo en el habla: el stress-timed 'ritmo acentual o de compás acentual' y el syllable-timed 'ritmo silábico o de compás silábico'. El ritmo acentual es característico de las lenguas germánicas, el inglés, el alemán, el holandés. La unidad rítmica es el pie acentual. Un pie acentual está constituido por una sílaba acentuada y, de izquierda a derecha, todas las sílabas inacentuadas hasta la próxima sílaba acentuada, que está excluida de ese pie acentual. 
El lapso temporal entre una sílaba acentuada y la siguiente sílaba acentuada tiende a ser constante, un efecto de isocronía acentual y de anisocronía silábica para conseguir distancias temporales similares con números de sílabas diferentes, esto es, una tendencia a realizar todos los pies acentuales con una similar duración. Para lograrlo, las sílabas más largas se comprimen y las sílabas más cortas se alargan. De esta manera se puede mantener una similaridad de la duración en los pies acentuales de diferente número de sílabas. Por ejemplo, en el inglés, hay una tendencia a reducir las sílabas inacentuadas tanto en duración como en frecuencia. Esta es una de las razones también de la presencia constante de vocales neutras, schwa. En cambio, el español tiende a un ritmo que está estrechamente relacionado con la sílaba y no con el pie acentual. Aquí, son las sílabas las que aparecen en intervalos más o menos similares. Emisiones con un número mayor de sílabas toman proporcionalmente más tiempo, y las sílabas no tienden a alargarse o a acortarse como en las lenguas germánicas. En este tipo de ritmo cada sílaba tiende a estar bien delineada. Este golpe rítmico de cada sílaba es recurrente y produce un efecto acompasado, aunque las sílabas acentuadas pueden ser más largas por el tipo de palabra (funcional o léxica), por razones contextuales y por razones suprasegmentales (ver también para los dos tipos de ritmo: Pointon, 1978, 1980; Faure et al., 1980; Major, 1981, 1983; Roach, 1982; Wenck y Wioland, 1982; Hoequist, 1983 a, 1983 b; Bertinetto, 1983; Dauer, 1983, 1987; Manrique y Signorini, 1983; Toledo, 1987, 1988 a; 1988 b, 1989, 1994, 1996, 1997, 1998; Bertinetto, 1989; Almeida, 1993, 1994; Almeida y Toledo, 1997; Ramus, 1999; Ramus et al., 1999; Frota y Vigário, 2001; Grabe y Low, 2002; Bertinetto y Bertini, 2007-2008, 2008; O'Rourke 2008; Arvaniti 2009).

Las dos tendencias, la isocronía silábica y anisocronía acentual y la isocronía acentual y la anisocronía silábica, se estudian experimentalmente. En español, Borzone de Manrique y Signorini (1983) indican que los pies acentuales poseen valores similares a los observados en lenguas germánicas. Toledo (1988 a) encuentra que las dos tendencias aparecen en diferentes corpus semiespontáneos tanto de laboratorio como discursos y textos leídos; asimismo, en el interior de los corpus. Dauer (1983, 1987) propone un nuevo enfoque: las tendencias rítmicas se basan en las diferencias fonológicas de las lenguas. Las lenguas que poseen reducción vocálica temporal y fonémica (el inglés, por ejemplo) y una fonotáctica compleja (tipos silábicos de hasta siete consonantes en el ataque y en la coda y de frecuencia de aparición muy relevante) se oponen a las lenguas sin reducción vocálica temporal y fonémica, sumado a una fonotáctica simple (un tipo silábico $\mathrm{CV}$, con una frecuencia de aparición muy alta), v. g., las lenguas germánicas se oponen a las lenguas romances, aunque se suman el ruso, el polaco y el árabe entre las lenguas de ritmo de isocronía acentual y el yoruba y el hindi entre las lenguas de ritmo de isocronía silábica (Ramus, 1999: 41). Dauer $(1983,1987)$ presenta un continuo con el inglés en el extremo y el español a distancia muy considerable de ese extremo inicial. Los hallazgos no son conclusivos; en cambio, son contradictorios. Parece que la señal acústica no puede reflejar las clase rítmica a la que pertenece una emisión. El fenómeno sería fundamentalmente perceptivo: los oyentes perciben que las sílabas acentuadas recaen a distancias casi equivalentes en las lenguas de isocronía acentual, que las sílabas acentuadas e inacentuadas tienen duraciones casi equivalentes en las lenguas de isocronía silábica y que las moras son de duración muy similar en las lenguas moraicas: el japonés, por ejemplo. 


\subsection{INFLUENCIAS FONOLÓGICAS}

\subsubsection{Los pies acentuales}

Las lenguas germánicas (isoacentuales, isocrónicas y anisosilábicas) presentan pies acentuales, entre un acento y el próximo excluido, de similar duración. Aunque la isocronía es imperfecta, las duraciones se reducen de manera inversamente proporcional al crecimiento del tamaño del pie ya sea sílabas o fonemas (fonos en la emisión). En la década de los 80 se realizan trabajos experimentales para probar el grado de isocronía acentual en lenguas germánicas y en lenguas romances, en el español. Dauer (1983) observa que las lenguas en general fluctúan entre 400 y 500 milisegundos (ms) en las duraciones de los pies. En español de Buenos Aires, Borzone de Manrique y Signorini (1983) obtienen resultados similares: los pies acentuales en español fluctúan entre promedios de 447 ms y 467 ms Toledo (1988 a: 77), también en español de Buenos Aires, analiza los datos de un texto fonético: la diferencia entre pies de 1 sílaba y 2 sílabas es de 1.58, una tendencia a la isocronía silábica, la diferencia entre pies de 4 sílabas y de 5 sílabas es de 1.19, una tendencia a la isocronía acentual. Dauer (1983) presenta resultados para el español equivalentes a Toledo (1988 a: 77). En inglés, Faure et al. (1980: 73) obtienen diferencias entre pies de 1 sílaba y de 2 sílabas de 1.62, una tendencia al isosilabismo, mientras que la diferencia entre pies de 4 sílabas y 5 sílabas es de 1.17, una tendencia a la isocronía acentual. En inglés, Uldall (1971) presenta resultados diametralmente opuestos a Faure et al. (1980: 73): tendencia a la isocronía acentual en los pies de pocas sílabas y un patrón isosilábico dentro de los pies de formato mayor ( 3 a 4 sílabas). Los hallazgos obtenidos no son coherentes tanto en las lenguas romances como en las lenguas germánicas. Las clases rítmicas no se separan claramente.

\subsubsection{Las sílabas}

La lengua española presenta sílabas abiertas CV con una frecuencia de aparición relativa del 51,35\% (Moreno Sandoval et al., 2006). En cambio, en inglés, la sílaba del tipo CV tiene una frecuencia de aparición relativa del 25,33\% (Gut y Milde, 2002). Las consonantes en el ataque y la coda (hasta 7 consonantes) forman una fonotáctica muy compleja. En español, la fonotáctica es de relativa simplicidad (hasta 4 consonantes).

\subsubsection{Las vocales}

Marín Gálvez (1994-95) analiza la duración de las vocales españolas. Encuentra seis factores que afectan la duración de las vocales: (1) la duración intrínseca de cada vocal, (2) el acento, (3) la estructura silábica, abierta o cerrada, (4) la sonoridad de la consonante posvocálica, (5) el modo de articulación de esa consonante posvocálica y (6) la posición de la vocal en la frase. La duración intrínseca indica este esquema según la mayor a la menor duración: $a>e>i>o>u$. La razón entre la vocal más larga [a] y la más corta [u] es de $1.14(14 \%)$. Este valor no es significativo con respecto a las just noticeable differentes, 'diferencias apenas perceptibles': la diferencia mínima para detectar un cambio temporal es del 35,9\% (Pamies y Fernández Planas, 2002). La 
diferencia entre vocales acentuadas y vocales inacentuadas es del 20\%, también bajo el nivel perceptivo. Las vocales en sílabas abiertas y en sílabas cerradas no presentan diferencias significativas (64.17 ms opuesto a 64.36, respectivamente). La sonoridad o la sordez de la consonante posvocálica no presenta diferencias significativas $(65.2 \mathrm{~ms}$ opuesto a $61.8 \mathrm{~ms}$, respectivamente). El modo de articulación de la consonante posvocálica no influye en la duración de las vocales. La posición prepausal alarga considerablemente las vocales, es $47 \%$ más larga que en posición interna (94.51 ms opuesto a $64.29 \mathrm{~ms}$, respectivamente). En suma: la duración vocálica es sensible a la posición en la frase. Se suman las diferencias observadas en el límite derecho de la frase fonológica $(\phi)$ : la sílaba final (la vocal final) se alarga considerablemente ante el tono de frase fonológica (T-, ya sea H- o L-); véase Prieto, 2006. Asimismo, la sílaba acentuada (la vocal acentuada) se alarga ante el tono de frase (T-); véase Rao, 2006, 2007; 2008; Toledo, 2008.

\subsection{MÉTRICAS RÍTMICAS}

\subsubsection{Ramus (1999), Ramus, Nespor y Mehler (1999), O’Rourke (2008 a)}

Ramus (1999) analiza las clases rítmicas de una manera nueva. Estudia las variaciones de intervalos vocálicos e intervalos consonánticos. Un intervalo vocálico es toda vocal, secuencia de vocales, sinalefas e hiatos y vocales agrupadas por resilabación en la cadena sintagmática. Un intervalo consonántico es toda consonante o grupo de consonantes entre intervalos vocálicos, también en la cadena sintagmática. Las pausas no cortan los intervalos, no se consideran. Se segmentan y se miden los intervalos vocálicos y consonánticos. Luego se calculan una serie de métricas rítmicas para determinar las clases de ritmo resultantes. Los intervalos vocálicos (IV) y los intervalos consonánticos (IC) son $/ E / \mathrm{ls} / a / \mathrm{x} / o / \mathrm{f} / o / \mathrm{ns} / e / \mathrm{t} / \mathrm{o} / \mathrm{c} / a / \mathrm{c} / o / \mathrm{n} / o / \mathrm{bs} / e / \mathrm{s} / i o ́ / \mathrm{h} /$, los IV se indican en itálica y los IC se indican en redonda. El análisis de las métricas rítmicas se basa en la propuesta de Dauer $(1983,1987)$ sobre las diferencias fonológicas que estructuran el ritmo de las lenguas universales, las germánicas, las romances y otras. Las lenguas que presentan reducción vocálica temporal y fonémica se separan de las lenguas que no presentan este rasgo; las lenguas que presentan una fonotáctica simple (tipos silábicos CV con alta frecuencia) se dividen de las lenguas con una fonotáctica compleja (por ejemplo, tipos silábicos de hasta 7 consonantes). Se calculan la proporcionalidad de intervalos vocálicos $(\% \mathrm{~V})$, también la desviación estándar de esos IV $(\Delta \mathrm{V}$ o Delta $\mathrm{V})$ y la desviación estándar de los intervalos consonánticos $(\Delta \mathrm{C}$ o Delta C). En la Figura 1 se muestran los resultados de dos métricas rítmicas ( $\% \mathrm{~V}$, los datos indicados por cuadrados no rellenos y Delta C, los datos indicados por cuadrados negros). La predicción es que las lenguas con un nivel de reducción temporal vocálica alto (fonémico) deberían tener una proporcionalidad de duración de intervalos vocáli$\cos (\% \mathrm{~V})$ bajos debido justamente a esa reducción; en cambio, la desviación estándar de la duración de intervalos consonánticos debería ser alta porque la fonotáctica es muy compleja y variada, y los tipos silábicos CV son de frecuencia de aparición baja (por ejemplo, en inglés, un 25,33\% (Gut y Milde, 2002). En la Figura 1, entonces, se pueden observar los datos de la proporcionalidad de la duración de intervalos vocálicos $(\% \mathrm{~V})$ en tres lenguas de isocronía o compás acentual (inglés, polaco y holandés): los valores son muy bajos, en ese orden, y se separan claramente de las lenguas de 
isocronía o compás silábico (francés, español, italiano y catalán): los valores son altos, en ese orden. El catalán no pertenece enteramente a una clase rítmica (Dauer, 1983; Nespor, 1990). El japonés, una lengua de ritmo moraico, muestra el valor más alto de proporcionalidad de la duración de intervalos vocálicos. La desviación estándar de la duración de intervalos consonánticos $(\Delta \mathrm{C}$ o Delta $\mathrm{C})$ presenta los valores más altos en las lenguas de isocronía o compás acentual; las lenguas de isocronía o compás silábico (francés, español, italiano y catalán) se agrupan en una banda del espacio. El japonés tiene el valor más bajo de desviación. Presenta una fuerte proporcionalidad de duraciones vocálicas y un mínimo de desviación con respecto a la media aritmética en la duración de los intervalos consonánticos. En suma: las lenguas se separan en clases: isocronía acentual, isocronía silábica y ritmo moraico (ver también: Ramus et al., 1999; para el español de Lima y Cuzco, ver O'Rourke, 2008 a).

Figura 1

Proporción de intervalos vocálicos $(\% \mathrm{~V})$ y desviación estándar de intervalos consonánticos (Delta $\mathrm{C}$ ) en ocho lenguas

(Ramus 1999; Ramus et al., 1999)

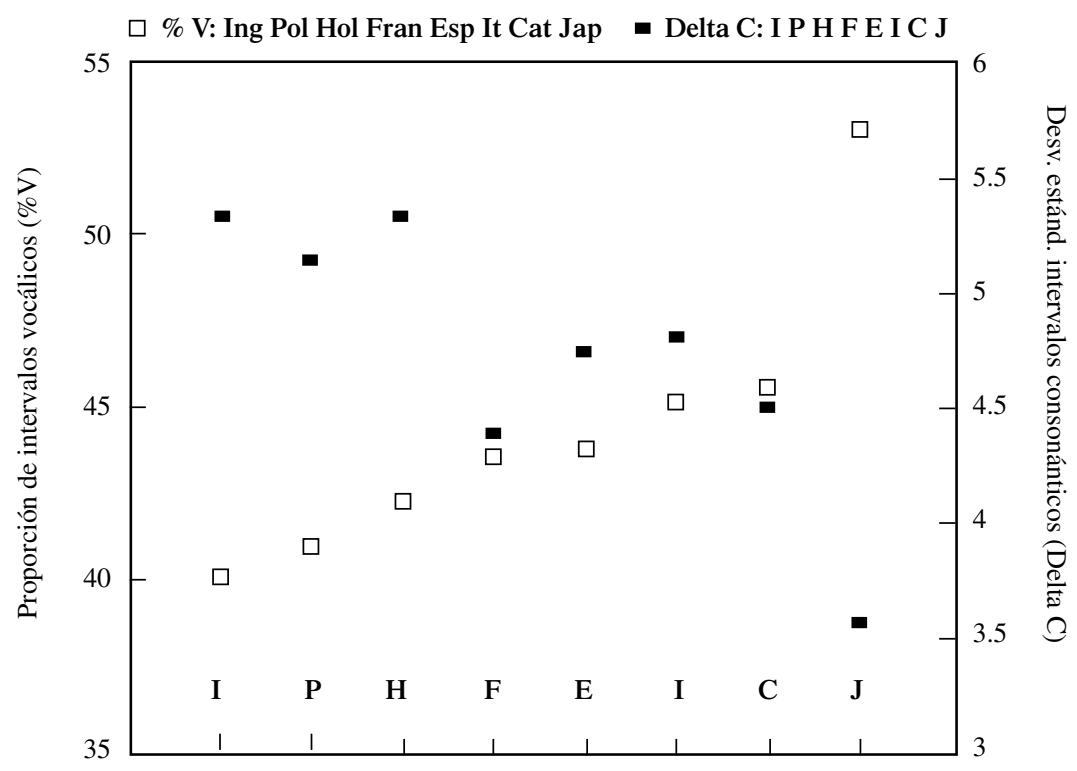

1.2.2. Grabe y Low (2002), Dellwo (2006); Dellwo (ms), White y Mattys, 2007, O’Rourke (2008 b)

Grabe y Low (2002), White y Mattys (2007) analizan las lenguas de compás silábico y de compás acentual. O'Rourke (2008 b) analiza el español de Lima y Cuzco. Se utilizan una serie de métricas nuevas: el Pairwise Variability Index 'variabilidad de pares sintagmáticos': los PVI-V (los pares de intervalos vocálicos) y los PVI-C (los pares de intervalos consonánticos). Se comparan los pares en forma sintagmática. Se predice que las dos métricas tengan valores inferiores en el español: 
la variabilidad de vocales es menos importante porque no hay reducción temporal vocálica (mayor isosilabismo) y la variabilidad consonántica es menor porque los tipos silábicos son más simples $(\mathrm{CV})$. En cambio, los valores más altos le pertenecen a las lenguas de compás acentual: las razones son opuestas a las de las lenguas de compás silábico: mayor reducción vocálica y mayor complejidad fonotáctica; por ejemplo, en el inglés. White y Mattys (2007) obtienen para el español una PVI-V de 36 y una PVI-C de 43 y para el inglés la PVI-V es de 73 y la PVI-C es de 70. Dellwo (2006) propone el Varco-V y el Varco-C. Se calcula el coeficiente entre la desviación estándar (de intervalos vocálicos y consonánticos) por la media aritmética de esos intervalos, vocálicos y consonánticos. En la fórmula se normalizan las diferencias de velocidad de habla entre los informantes, un factor fundamental en el análisis (ver más adelante). En el español se observa un valor de 41 (intervalos vocálicos) y de 46 (intervalos consonánticos). En el inglés se observan los valores más altos: 64 en intervalos vocálicos y 47 en intervalos consonánticos. Aquí, en el Varco-C, no hay contrastes tan netos como en el Varco-V. O'Rourke (2008 b) obtiene valores muy coherentes para el español peruano. Los hallazgos en los corpus de Lima tienen valores de 39, en el Varco-V, y 37, en el Varco-C. Toledo (enviado) obtiene valores similares en el español de Sevilla.

En la Figura 2 se observan los valores obtenidos por White y Mattys (2007) en español, francés, inglés y holandés, dos lenguas de isocronía silábica y dos lenguas de isocronía acentual. Se muestran las métricas rítmicas Varco-V (el coeficiente de variabilidad de intervalos vocálicos), la variabilidad de pares vocálicos, sintagmáticos (PVI-V), normalizado (ver la fórmula más abajo), el Varco-C (el coeficiente de variabilidad de intervalos consonánticos) y la variabilidad de pares consonánticos en cálculo sintagmático (PVI-C), no normalizado (ver la fórmula más abajo). Se confrontan con los resultados obtenidos por Toledo (enviado) sobre un corpus de Sevilla. A la izquierda de la Figura, el Varco-V se señala por soles (White y Mattys, 2007), el Varco-V en el español sevillano se indica por las casas no rellenas (Toledo, enviado). El PVI-V se señala por asteriscos (White y Mattys, 2007), el PVI-V en el español de Sevilla se indica por cuadrados no rellenos (Toledo, enviado). A la derecha de la Figura, se presenta el Varco-C y se señala por rombos negros (White y Mattys, 2007), el Varco-C del español sevillano se indica por cruces (Toledo, enviado). El PVI-C se señala por círculos negros (White y Mattys, 2007), el PVI-C del español de Sevilla se indica por el símbolo de la libra (Toledo, enviado). Se ven claramente las tendencias rítmicas: el español en White y Mattys (2007) se acerca a los valores obtenidos por Toledo (enviado) en el español de Sevilla, los valores más bajos en las cuatro métricas rítmicas. Los valores más altos pertenecen a las lenguas de compás acentual (inglés y holandés). El Varco-C muestra algunas incoherencias en White y Mattys (2007), no así en los datos de Toledo (enviado). En síntesis: la baja reducción temporal vocálica de una lengua y una fonotáctica simple (tipos silábicos CV) evitan la variabilidad de los intervalos vocálicos y consonánticos; de este modo, las lenguas se agrupan en clases rítmicas muy claras. Las lenguas afines se insertan en bandas de valores similares o próximos, y las lenguas alejadas fonológicamente ocupan bandas opuestas. 
Figura 2

Valores de Varco-V, PVI-V, Varco-C y PVI-C en español, francés, inglés y holandés (White y Mattys, 2007) y en Amper-Sevilla (Toledo, en prensa)

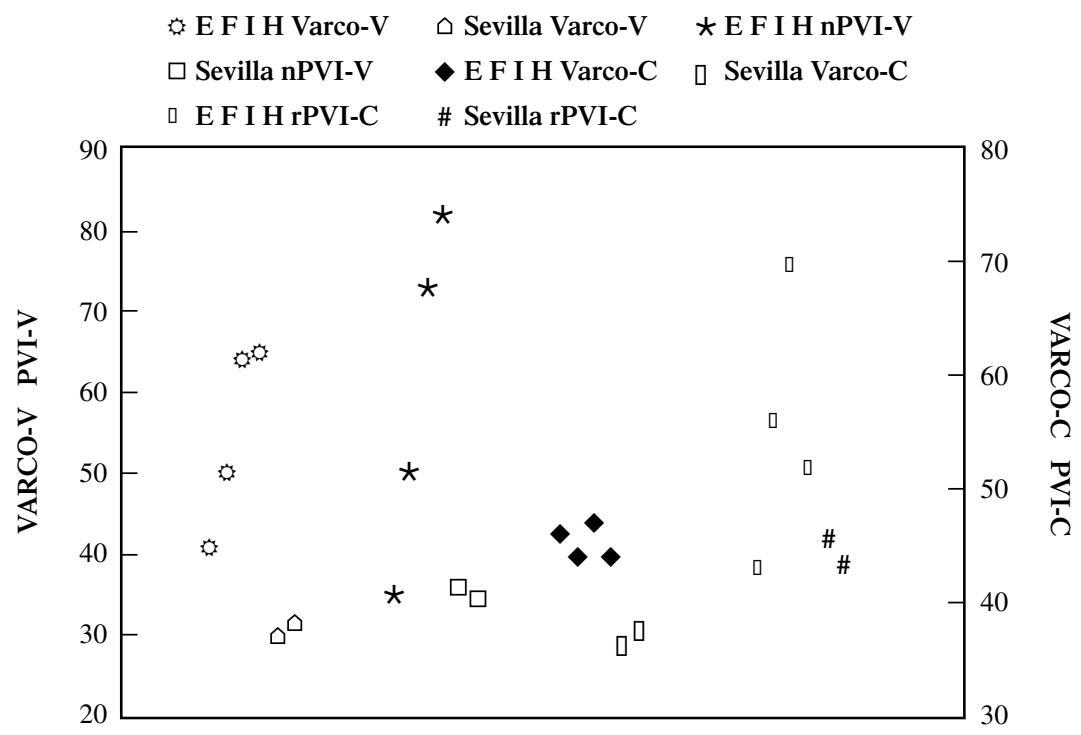

\subsubsection{Normalización de las métricas rítmicas}

Dellwo (ms) estudia el problema de las imperfecciones de los cálculos debidas a los cambios de la velocidad de habla en cada Informante y en las emisiones de cada Informante a lo largo de las emisiones. Propone una métrica que no presenta perturbaciones debidas a las diferencias de velocidad de habla: la proporcionalidad de intervalos vocálicos $(\% \mathrm{~V})$. Analiza una métrica normalizada en que se soluciona este problema del cálculo en intervalos vocálicos: nPVI-V (ver White y Mattys, 2007; O'Rourke, 2008 b, entre otros). Por último, sugiere dos métricas normalizadas para los cálculos de intervalos consonánticos: nPVI-C y la $\mathrm{n} \log$ Delta $\mathrm{C}(\mathrm{n} \log \Delta \mathrm{C})$, en este último caso se realiza una normalización logarítmica (ver la explicación y las fórmulas más adelante).

\section{Metodología}

\subsection{CORPUS E INFORMANTES}

Se analizan tres muestras del corpus Amper-España ${ }^{1}$. Se eligen tres muestras similares (Amper-Aragón, Amper-Canarias y Amper-Granada). Los materiales están integrados por oraciones declarativas SVO: SN + SV + SPrep. Los SN son palabras

\footnotetext{
${ }^{1}$ Agradezco muchísimo el permiso para la utilización de las muestras elegidas de Amper-España. Vaya mi agradecimiento al Dr. Javier Simón Casas (Amper-Aragón), a la Dra. Josefa Dorta (Amper-Canarias) y al Dr. Antonio Pamies Bertrán (Amper-Granada).
} 
oxítonas, paroxítonas y proparoxítonas (saxofón, guitarra y cítara). Los SPrep también se realizan en palabras con los tres tipos de acentos léxicos: con obsesión, con paciencia, con pánico. Los SV son paroxítonos: se toca. Las oraciones, entonces, son del tipo La guitarra se toca con paciencia, con un fraseo fonológico (entonativo) estándar $[(\mathrm{SN}) \phi$ ip (SV + SPrep) $\phi$ ip IP] ( $\phi$ es frase fonológica; ip es frase entonativa intermedia; IP es frase entonativa mayor), aunque se observan variaciones en el fraseo y en los tonos de frase (H-, L-, altos y bajos, respectivamente). Las oraciones son 9 x 3 Informantes, 1 por dialecto. La grabación se produce según la técnica de elicitation task 'entrevista dirigida': cada Informante responde a preguntas, con oraciones declarativas inducidas. En Aragón se elige un dialecto de la ciudad de Jaca, en los Pirineos; en las Islas Canarias, el Informante es de Tenerife; en Granada, el Informante es urbano, pero con origen y una existencia prolongada en la sierra, en el ámbito rural. Los tres Informantes son femeninos, de edad adulta, sin estudios superiores (Ver para el Amper-Aragón: Simón Casas, 2007: 561-570, Simón Casas, 2008; para el Amper-Canarias: Dorta y Hernández Díaz, 2005; para el Amper-Granada: Pamies y Amorós, 2005).

\subsection{ANÁLISIS ACÚSTICO Y SEGMENTACIÓN DE INTERVALOS VOCÁLICOS Y CONSONÁNTICOS}

El material seleccionado se analiza por medio del programa Speech Analyzer 3.0.1 (Summer Institute of Linguistics). La mediciones de la duración se estudian en la forma de onda, en el espectrograma y en el contorno de la fundamental, en la entonación. Las tres visualizaciones se superponen y cooperan en la decisión. En las Figuras 3, 4 y 5 se observan fragmentos de las oraciones. En la Figura 3 el fragmento pertenece a la oración El saxofón se toca con obsesión. La segmentación de los intervalos consonánticos y los intervalos vocálicos se indica por líneas verticales; en este caso, en la forma de onda (arriba) y en el espectrograma (debajo). Se considera que un intervalo vocálico es una vocal o un grupo de vocales, sin consideración de las pausas posibles entre palabras. Los diptongos son parte de un intervalo vocálico, como grupo de vocales (Massone y Borzone de Marique, 1985: 102-103). En otras lenguas se considera que los diptongos crecientes (paciencia) son parte del intervalo consonántico anterior, y los diptongos decrecientes (reina) son parte del intervalo vocálico (Ramus, 1999; Frota y Vigário, 2001; White y Mattys, 2007; O’Rourke, 2008 a, 2008 b). En la Figura 3 se puede observar que los intervalos vocálicos tienen duraciones similares, el diptongo se aproxima al doble de una vocal. La vocal acentuada en el SV no presenta duraciones por influencia suprasegmental. Tampoco el diptongo en posición final absoluta. Los intervalos consonánticos tienen duraciones según el tipo de consonante: las fricativas $>$ oclusivas $>$ nasales. Se pronuncia el grupo culto (obsesión), oclusiva más fricativa.

En la Figura 4 se observan la forma de onda y el espectrograma en la muestra del español de Tenerife. La oración es La cítara se toca con pánico. El fragmento está segmentado en intervalos vocálicos y consonánticos. Las vocales tienen duraciones similares. El acento puede influir en la duración (toca) o no hacerlo (cítara). Las consonantes presentan un esquema estándar de duraciones: fricativas $>$ oclusivas $>$ vibrantes simples. La vibración de las cuerdas vocales puede continuar en los tramos sordos (se toca), pero es parte del intervalo consonántico. 
Figura 3

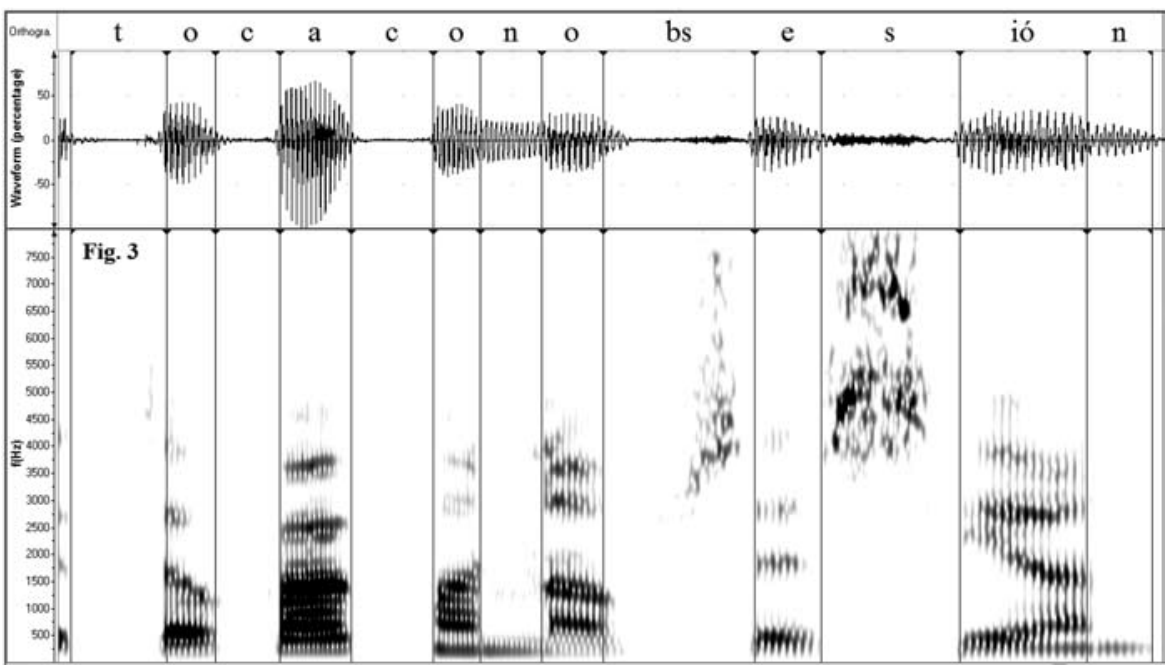

Figura 4

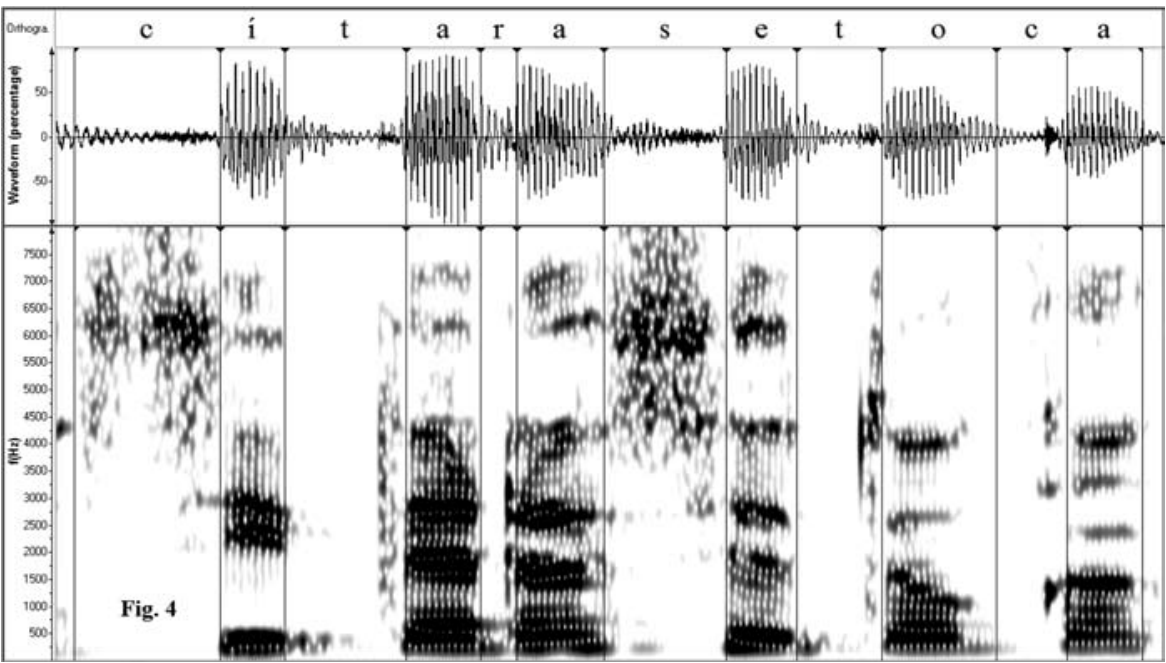

En la Figura 5 se puede visualizar el fragmento de la Informante de Granada, dialecto urbano con origen y estadía prolongada en el ámbito serrano, rural. El fragmento está segmentado también en intervalos vocálicos e intervalos consonánticos. Se observan elisiones de vocales ( $s^{\prime} t o$ ). En el mismo fragmento se pueden observar elisiones completas de sílabas, con alargamiento temporal de la vocal y la oclusiva en la sílaba acentuada (to'con). La duración de los intervalos consonánticos varía y también la duración de los intervalos vocálicos. No hay influencia acentual sobre una vocal (cítara). En cuanto a las consonantes, el esquema es fricativas $>$ laterales 
$>$ oclusivas $>$ vibrantes simples. La velocidad de habla de esta Informante es de 7.03 sil. por segundo. Es similar a la velocidad de habla de la Informante de Tenerife (6.88 sil/seg.) y también es similar a la velocidad de habla de la Informante de Aragón (6.72 sil/seg.). A pesar de ello, se controla la velocidad en los cálculos por medio de normalizaciones especiales (Dellwo, ms).

Figura 5

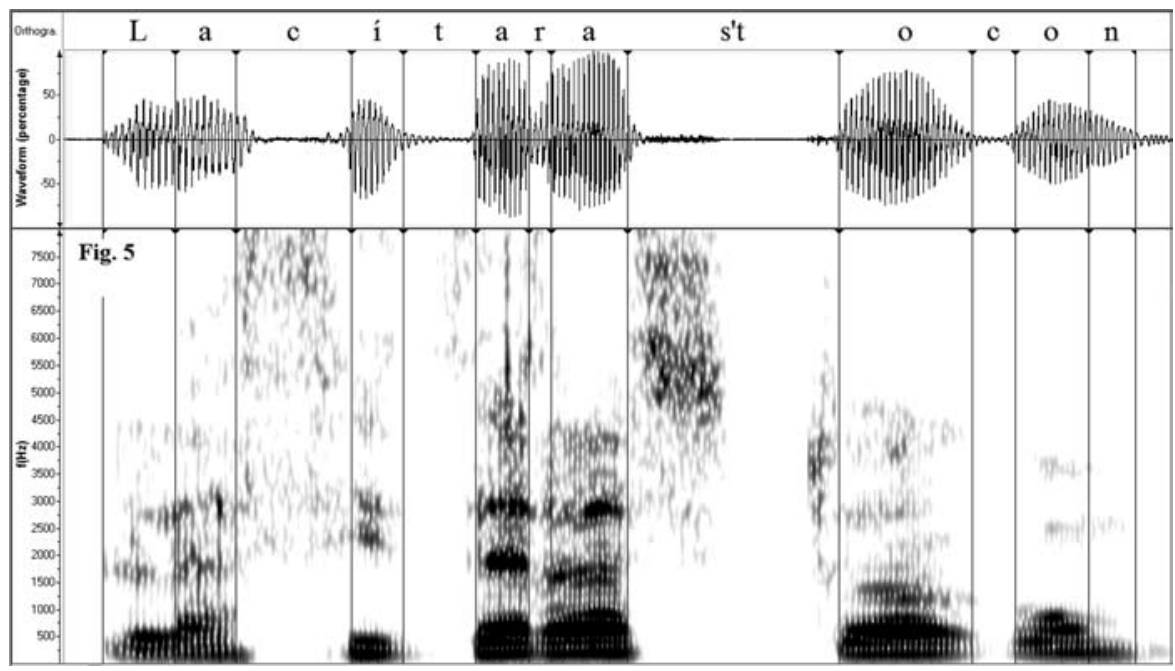

\subsection{MÉTRICAS RÍTMICAS ELEGIDAS}

Se calculan dos métricas rítmicas para los intervalos vocálicos. La \%V es el cociente entre la medición global de los intervalos vocálicos y la duración total de cada oración. Se multiplica por 100 para facilitar la lectura (ver arriba). Este cálculo no está influido por los cambios de la velocidad de habla en el transcurso de la producción de un Informante y entre Informantes (Dellwo, ms). La predicción es que en una lengua de isocronía silábica debería tener valores más altos de proporcionalidad vocálica, que en una lengua de isocronía acentual (ver Figura 1; también Ramus, 1999; Ramus et al., 1999; Frota y Vigário, 2001; White y Mattys, 2007; O’Rourke, 2008 a; Toledo et al., 2009). Asimismo, se calcula una métrica rítmica normalizada para evitar las diferencias de velocidad de habla, la n PVI-V: Normalised Paiwise Variability Index, 'índice de intervalos vocálicos adyacentes en la cadena sintagmática'; se calcula la suma de las diferencias de los pares de intervalos vocálicos, se normaliza por medio del cociente de cada diferencia por la media aritmética de los dos intervalos vocálicos en cuestión; se calcula el cociente de la suma de los pares normalizados por $n$ pares- 1 . Luego se multiplica por 100 para facilitar la lectura y la comparación con otros trabajos anteriores (Grabe y Low, 2002; White y Mattys, 2007; O'Rourke, 2008 b); Gurlekian et al., 2009). La fórmula del Paiwise Variability Index (normalizado) es nPVI-V $=\sum$ $(((\mathrm{d} a-\mathrm{d} b) \div($ media $\mathrm{d} a$ y $\mathrm{d} b) \div n-1) \times 100$. Dellwo $(\mathrm{ms})$ indica que esta métrica rítmica no está influida por los cambios de velocidad de habla. Se calculan dos métricas para 
el control de los intervalos consonánticos propuestas por Dellwo (ms). La primera es la $\mathrm{n} \log$ (base 2, natural) Delta $\mathrm{C}$, se transforma cada dato por el logaritmo con base e, luego se calcula la desviación estándar (Delta $\mathrm{C}, \Delta \mathrm{C}$ ). El resultado queda liberado de la deformación por los cambios de velocidades de habla. Así, las duraciones breves debidas a velocidades rápidas se aproximan a las duraciones largas debidas a velocidades lentas. Se obtiene una variación proporcional y no las diferencias de valores absolutos. La segunda métrica es la n PVI-C, el índice de variabilidad entre pares consonánticos enfrentados en la cadena sintagmática. La diferencia con trabajos anteriores (Grabe y Low, 2002; White y Mattys, 2007; O'Rourke, 2008 b; Gurlekian et al., 2009, entre otros) es que la métrica está normalizada de manera similar que en la propuesta de la $\mathrm{n}$ PVI-V. La fórmula del Paiwise Variability Index (normalizado) es n PVI-C $=\sum(((\mathrm{d} a-\mathrm{d} b) \div($ media $\mathrm{d} a \mathrm{y} \mathrm{d} b) \div n-1) \times 100$. Como en los intervalos vocálicos, se calcula la suma de las diferencias de los pares de intervalos consonánticos, se normaliza por medio del cociente de cada diferencia por la media aritmética de los dos intervalos consonánticos enfrentados en la cadena; se calcula el cociente de la suma de los pares normalizados por $n$ pares-1. Luego se multiplica por 100 para facilitar la lectura. Dellwo (ms) demuestra que esta métrica, normalizada de este modo, no sufre influencias por los cambios de velocidad de habla. En este trabajo sobre tres dialectos de Amper-España se utiliza como métrica de control. En suma: se analizan las cuatro métricas normalizadas en tres muestras de Amper-España: Amper-Aragón (Jaca), en Amper-Canarias (Tenerife) y en Amper-Granada.

\section{ANÁLISIS DE LAS MÉTRICAS RÍTMICAS CALCULADAS}

Figura 6

Valores en Amper-Sevilla (Toledo, enviado), Amper-Argentina (Toledo et al., 2009), Lima y Cuzco (O'Rourke, 2008a), Amper-Aragón, Amper-Canarias y Amper-Granada

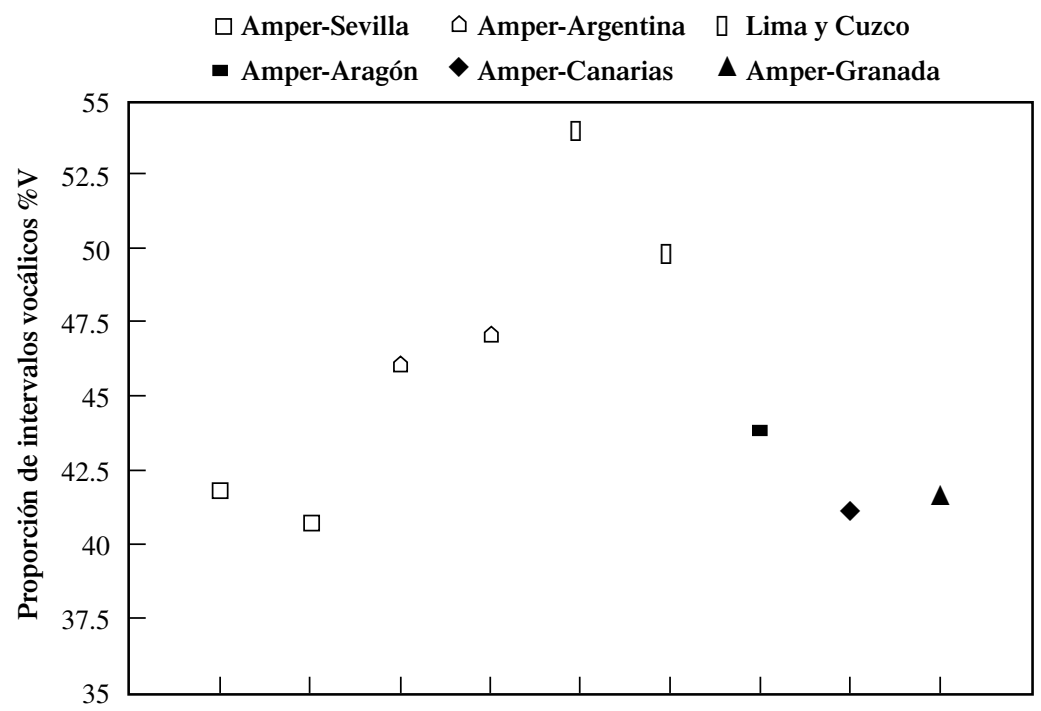


En la Figura 6 se muestran los cálculos obtenidos por medio de la métrica rítmica $\% \mathrm{~V}$, la proporcionalidad de las duraciones de los intervalos vocálicos, en las tres muestras de Amper-España. En Amper-Aragón (la zona de Jaca, en los Pirineos) el valor obtenido es 43.83; en Amper-Canarias (Tenerife) el valor es 41.1; en AmperGranada el resultado es 41.54. Los tres valores son próximos, indican un grado de proporcionalidad de la duración de los intervalos vocálicos que reflejan algún grado de reducción vocálica. De forma similar, en Amper-Sevilla (Toledo, enviado) también se encuentra un valor similar en los dos Informantes analizados, es 41.78 y 40.68 . Los resultados muestran un grado de reducción vocálica en los intervalos vocálicos. En cambio, los hallazgos en Hispanoamérica muestran una proporcionalidad de las duraciones en los intervalos vocálicos más alta: Amper-Argentina (46.02 en Informantes Instruidos (universitarios), 47 en Informantes No Instruidos, Toledo et al., 2009); en Lima (54), en Cuzco (49.6), ver O'Rourke (2008 a). Estos resultados sugieren que los Informantes españoles producen reducciones más relevantes que los Informantes hispanoamericanos, por lo menos en las muestras estudiadas.

Figura 7

Valores de nPVI-V en español, francés, inglés y holandés (White y Mattys, 2007), en Amper-Sevilla (enviado), en Amper-Argentina (Gurlekian et al., 2009) y en Lima y Cuzco (O'Rourke, 2008b), Amper-Aragón, Amper-Canarias y Amper-Granada

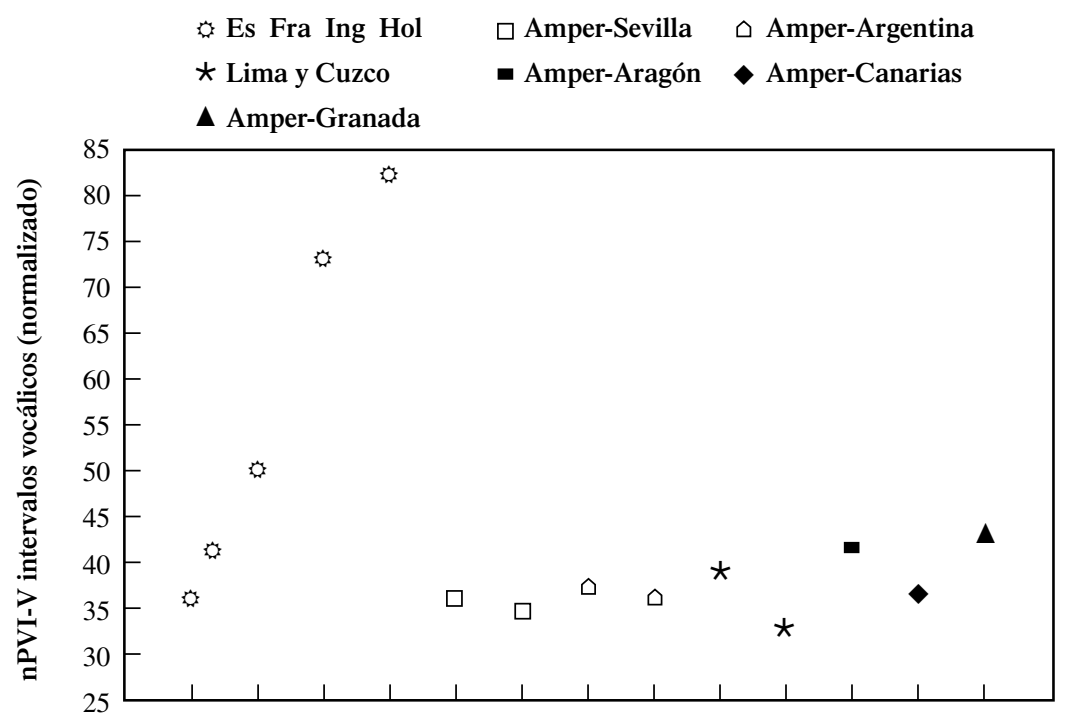

En la Figura 7 se presentan los resultados obtenidos sobre los intervalos vocálicos relacionados en la cadena sintagmática (Grabe y Low, 2002), la métrica rítmica está normalizada (ya explicada arriba). Se predice que las lenguas de isocronía acentual (las lenguas germánicas, entre otras) tendrían valores más altos que las lenguas de isocronía silábica (las lenguas romances, entre otras) debido a la fonotáctica simple y a la reducción vocálica débil. Efectivamente, el primer valor de White y Mattys (2007) es el español (36) y, en escala ascendente, el francés, el inglés y el holandés $(50,73,82$, respectivamente). El francés es una lengua de isocronía silábica, pero 
con mayor grado de variabilidad de las duraciones de intervalos vocálicos que el español. Tanto los dialectos peninsulares y el dialecto insular como los dialectos hispanoamericanos se insertan en una banda de valores bajos, perfectamente incrustados en su clase rítmica, de isocronía silábica. Amper-Sevilla tiene valores de 36.07 y 34.76 en sus dos Informantes (Toledo, enviado), Amper-Argentina tiene valores de 36.63 y 36.14 en sus resultados de los grupos Instruidos y No Instruidos (Gurlekian et al., 2009; Lima tiene un valor de 39 y Cuzco presenta un valor de 33 (O'Rourke, 2008 b). Amper-Aragón tiene un valor de 41.54; en Amper-Canarias (Tenerife) se observa un resultado de 36.49; Amper-Granada tiene un valor de 42.56. El mayor grado de variabilidad le corresponde a Amper-Granada: la Informante vive en la zona urbana, pero es de origen serrano, rural. Presenta reducciones y elisiones vocálicas (ver Figura 5). La menor variabilidad le corresponde a la Informante de Tenerife; los intervalos vocálicos tienden a duraciones estándar, aunque influidos por razones de acento, moderadamente, y entonación; en este caso, en la Figura, por tonos de frontera intermedia (Ver Figura 4).

Figura 8

Valores de $\mathrm{n} \log$ (base e) Delta C Amper-Aragón, Amper-Canarias y Amper-Granada comparados con Delta C (no normalizados) en Amper-Sevilla (enviado), en AmperArgentina (Toledo et al., 2009) y en Lima y Cuzco (O'Rourke, 2008a)

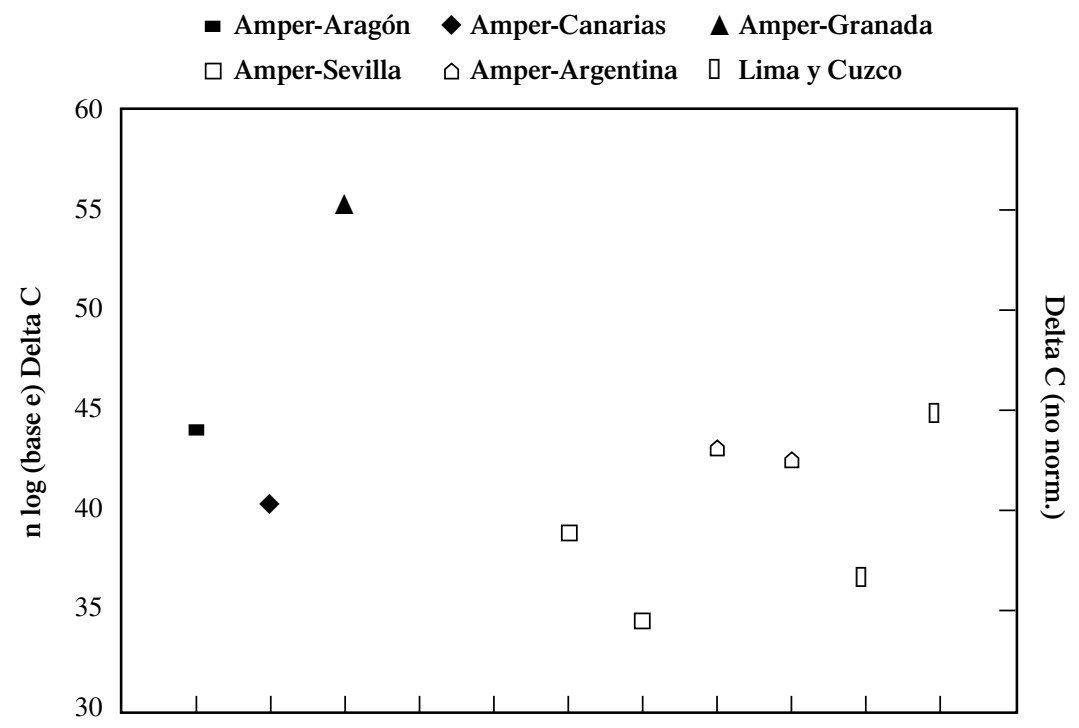

En la Figura 8 se muestran los resultados obtenidos con la métrica rítmica $\mathrm{n}$ log (base e) Delta C, es decir, las desviaciones estándar de los intervalos consonánticos en Amper-Aragón, en Amper-Canarias y en Amper-Granada. Los datos naturales se transforman en logaritmos de base e, luego se calcula la desviación estándar, Delta C (Dellwo, ms). Los resultados normalizados se observan en la parte izquierda de la Figura. En la parte derecha de la Figura, sólo a manera de comparación, con reparos, se observan los datos obtenidos en trabajos anteriores, no normalizados: en Amper- 
Sevilla (Toledo, enviado), en Amper-Argentina (Toledo et al., 2009), en Lima y Cuzco (O'Rourke, 2008 a). Los datos en Aragón, Tenerife y Granada son los siguientes: $44.13,40.3$ y 55.14, respectivamente. Estos datos se asemejan a los obtenidos, pero no normalizados, en Sevilla (38.94 y 34.60, dos Informantes), en Argentina (42.94 y 42.48, Informantes Instruidos y No Instruidos, respectivamente) y en Lima y Cuzco (36.7 y 45, respectivamente. En los datos de este trabajo, Amper-Granada presenta el nivel más alto de desviación de los intervalos consonánticos. Como puede observarse en la Figura 5, la Informante produce elisiones drásticas de sílabas. El fenómeno prosódico se explica por el origen y la permanencia prolongada en ambiente rural, aunque en la actualidad su medio sea urbano.

Por último, se calcula una métrica rítmica de control y normalizada, el índice de variabilidad de intervalos consonánticos, en pares y normalizados por la media de esos pares en la cadena sintagmática, el n PVI-C (ver la fórmula más arriba). Es otra de las métricas propuestas por Dellwo (ms) que está liberada de las fluctuaciones de la velocidad de habla en la emisión. Los datos son los que siguen: Aragón (53.54), Tenerife (39.2) y Granada (68.91). Tenerife resulta, como en la n log (base e) Delta C, con el nivel más bajo de variabilidad de intervalos consonánticos (ver Figura 4). Granada presenta el nivel más alto de variabilidad de intervalos consonánticos (por las razones ya explicadas, ver Figura 5).

\section{CONCLUSIÓN}

Los resultados obtenidos en este trabajo confirman que el español como lengua romance pertenece a la clase rítmica de lenguas de isocronía silábica o anisocronía acentual, isosilábicas o de compás silábico opuestas a las lenguas de isocronía acentual y anisocronía silábica, anisosilábicas o de compás acentual, esto es, las lenguas germánicas, entre otras. La confirmación se obtiene en la señal acústica que refleja las diferencias fonológicas entre esas lenguas. Así, se separan las lenguas de fonotáctica simple (tipos silábicos $\mathrm{CV}$, con un alto grado de frecuencia de aparición) y la ausencia de reducción temporal vocálica y fonémica y las lenguas con fototáctica compleja (tipos silábicos con un número considerable de consonantes en el ataque y en la coda, con frecuencia de aparición relevante), es decir, las lenguas romances opuestas a las lenguas germánicas. Para observar esta oposición y también con el fin de fijar la clase rítmica de cada lengua, se calculan métricas rítmicas. Al hacerlo, se tienen en cuenta las perturbaciones de duración producidas en las emisiones por la influencia de los cambios en la velocidad de habla. Para ello, se normalizan cuatro métricas rítmicas en que se controla perfectamente este efecto de la velocidad de habla. Los materiales son sometidos a una segmentación en intervalos vocálicos (todo grupo de vocales en secuencia) e intervalos consonánticos (unión de consonantes en continuidad sintagmática). En la primera métrica, se busca la proporcionalidad de los intervalos vocálicos en las secuencia $(\% \mathrm{~V})$ : el cociente de la suma de los intervalos vocálicos y la duración total de la secuencia. La predicción sobre los valores más altos en el español por la ausencia de reducción vocálica fonémica se cumple. El español se agrupa en su clase rítmica. En la segunda métrica, se intenta encontrar un índice de variabilidad de intervalos vocálicos en pares (n PVI-V). Se predice que el español podría tener valores más bajos con respecto a las lenguas que no pertenecen 
a su clase rítmica por la tendencia al isosilabismo. Efectivamente, los resultados obtenidos por medio de esta métrica confirman la pertenencia del español a su clase rítmica. En la tercera métrica se estudian los intervalos consonánticos por medio de la desviación estándar de las duraciones (normalizadas logarítmicamente: $\mathrm{n}$ log (base e) Delta C). Se predice que una lengua de fonotáctica simple como el español tendría desviaciones estándar más bajas que las lenguas con fototácticas más complejas, las lenguas germánicas, entre otras. Los hallazgos confirman esta predicción para las muestras de Amper-España elegidas. Se calcula una cuarta métrica, a manera de control. Se logra un índice de variabilidad de los intervalos consonánticos en pares (n PVI-C). Se predice una variabilidad entre pares menor en el español, si se lo compara a otras lenguas con fonotácticas complejas. Los resultados confirman esta tendencia del español. La lengua se inserta convenientemente dentro de su clase rítmica. Se observa, además, que los dialectos elegidos en el Amper-España tienen diferencias provocadas por situación sociolectal. Las Informantes de regiones urbanas (Tenerife, en Canarias > Jaca, en Aragón, en este orden) son más cuidados en sus producciones que la Informante de origen serrano, rural (Granada). Las métricas son sensibles a la variación temporal producida por reducción consonántica y elisión silábica.

\section{Discusión}

Los resultados obtenidos en este trabajo confirman, en cierta medida, lo propuesto por Ramus (1999) y Ramus et al. (1999) en cuanto a la proporcionalidad de intervalos vocálicos para la clase rítmica de las lenguas romances (entre ellas, el español), la clase rítmica de las lenguas germánicas, entre otras, y el japonés. El español tiene un alto grado de proporcionalidad de intervalos vocálicos con respecto a los intervalos consonánticos. Sin embargo, este resultado es sensible a los cambios dialectales y sociolectales dentro de la clase (ver Toledo et al., 2009; Toledo, enviado y este estudio sobre muestras del Amper-España). Se confirman los hallazgos en White y Mattys, 2007, en español, francés, inglés y holandés. El español tiene el grado mayor de proporcionalidad de duración de los intervalos vocálicos; asimismo, tiene la desviación estándar menor de intervalos vocálicos, aunque no estén normalizados como en este trabajo sobre muestras del Amper-España. La variabilidad de intervalos vocálicos en pares, normalizada, es menor en español (White y Mattys, 2007) similar a este trabajo.

De igual modo, estos resultados sobre los tres dialectos del Amper-España confirman hallazgos anteriores obtenidos en muestras de Amper-Argentina (Informantes con estudios superiores, graduados, comparados con Informantes sin estudios superiores). El español queda perfectamente inmerso en su clase rítmica (Toledo, et al., 2009; Gurlekian et al., 2009). Son también similares a los obtenidos en Lima y Cuzco por O'Rourke (2008 a, 2008 b). Se confirma, además, lo observado en una muestra de Amper-Sevilla con métricas rítmicas similares, pero no equivalentes (el grado de normalización en este estudio sobre los tres dialectos Amper-España es global (las cuatro métricas rítmicas propuestas por Dellwo, ms), pero es parcial en Amper-Sevilla). En este estudio se abre la posibilidad de estudios de la variación rítmica por diferencias geográficas (áreas de Hispanoamérica no estudiadas), por diferencias sociolectales y por diferencias estilísticas. 


\section{OBRAS CITADAS}

Abercrombie, David. 1967. Elements of General Phonetics. Edimburgo: Edinburgh University Press.

Almeida. Manuel. 1993. "Alternancia temporal y ritmo en español”. Verba 20. 433-443. 1994. "Patrones rítmicos del español: Isocronía y alternancia". Estudios Filológicos 29. 7-14.

— y Guillermo Toledo. 1997. "Alternancia del ritmo en español”. Contribuciones al estudio de la lingüística hispánica. Coord. Manuel Almeida y Josefa Dorta. Santa Cruz de Tenerife: Cabildo de Tenerife/Montesinos. 35-42.

Arvaniti, Amalia. 2009. "Rhythm, timing and the timing of rhythm". Phonetica 66: 46-63.

Bertinetto, Pier Marco. 1989. "Reflections on the dichotomy 'stress-vs. syllable-timing". Revue de Phonétique Appliquée 91-92-93. 99-130.

_ y Chiara Bertini. 2007-2008. "Towards a unified predictive model of natural language rhythm". Quaderni del Laboratorio di Linguistica 7. s. p.

— y Chiara Bertini. 2008. "On modelling the rhythm of natural languages". Proceedings. of Speech Prosody 2008, Fourth Conference on Speech Prosody. Eds. Plinio A. Barbosa, Sandra Madureira y César Reis. Campinas: IEL, Unicamp, Motorola, Editora RG. Edición en CD-ROM.

Borzone de Manrique, Ana María y Angela Signorini. 1983. "Segmental duration and rhythm in Spanish". Journal of Phonetics 11. 117-128.

Dauer, Rebecca. 1983. "Stress-timing and syllable-timing reanalized". Journal of Phonetics 11. 51-62.

- 1987. "Phonetic and phonological components of language rhythm". Proceedings of the XIth International Congress of Phonetic Sciences 5. Tallinn. 447-450.

Dellwo, Volker. 2006. "Rhythm and speech rate: a variation coefficient for delta C". Sprache und Sprachverarbeitung: Akten des 38. Linguistischen Kolloquiums in Piliscsaba 2003/ Language and language-processing: Proceedings of the 38th Linguistics Colloquium, Piliscsaba 2003, Linguistik International. Eds. Pawe Karnowski e Imre Szigeti. Frankfurt am Main: Peter Lang Publishing Group. 231-241.

rhythm".

Dorta, Josefa. 2008. "Base de Datos Amper-Can”. En línea: www.ull.es /users/ labfon/ proampercan/index.html.

—_ y Beatriz Hernández Díaz. 2004. "Prosodia de las oraciones SVO declarativas e interrogativas en el español de Tenerife”. Estudios de Fonética Experimental XIII. 225-273.

Faure, Georges, Daniel Hirst y Michel Chafcouloff. 1980. "Rhythm in English: Isochronism, pitch, and perceived stress", The Melody of Language. Eds. Linda R. Waugh y C. H. van Schooneveld. Baltimore: University Park Press. 71-79.

Fernández Planas, Ana. 2005. "Aspectos generales acerca de proyecto internacional AMPER en España". Estudios de Fonética Experimental XIV. 13-27.

Frota, Sónia y Marina Vigário. 2001. "On the correlates of rhythmic distinctions: The European/ Brazilian Portuguese case”. Probus 13. 247-275.

Grabe, Esther y Ee Ling Low. 2002. "Durational variability in speech and the rhythm class hypothesis". Papers in Laboratory Phonology 7. Eds. Carlos Gussenhoven y Natasha Warner. Berlín: Mouton. 515-546.

Gurlekian, Jorge, Reina Yanagida, Mónica Trípodi y Guillermo Toledo. 2009. “Amper-Argentina: variabilidad rítmica en dos corpus". Comunicación en las V Jornadas Internacionales de Educación Lingüística: Lenguaje y Comunicación, Realidades y Desafíos, Entre Ríos, Argentina. 
Gut, Ulrike y Jan-Torsten Milde. 2002. "The prosody of Nigerian English”. Speech Prosody 2002. Eds. Bernard Bel e Isabelle Marlien. 367-370.

Hoequist, Charles. 1983 a. "Durational correlates of linguistic rhythm categories". Phonetica 40. 19-23.

. 1983b. "Syllable duration in stress-, sylable- and mora-timed languages". Phonetica 40. 202-237.

Major, Roy. 1981. "Stress-timing in Brazilian Portuguese" . Journal of Phonetics 9. 342-351.

—. 1985. "Stress and rhythm in Brazilian Portuguese". Language 61. 259-282.

Marín Gálvez, Rafael. 1994-1995. "La duración vocálica en español”. E.L.U.A. Estudios de Lingüística, Universidad de Alicante. 213-226.

Martínez Celdrán, E. y Ana Fernández Planas. 2005. "Estudio metodológico acerca de la obtención del corpus fijo en el proyecto Amper". Estudios de Fonética Experimental XIV. 29-66.

Massone, María I. y Ana María Borzone de Manrique. 1985. Principios de transcripción fonética, Buenos Aires: Ediciones Macchi.

Nespor, Marina. 1990. "On the rhythm parameter in phonology". Logical issues in Language Acquisition I. Ed. Iggi Roca. Dordrecht: Foris. 157-175.

O'Rourke, Erin. 2008. "Correlating speech rhythm in Spanish: Evidence from two Peruvian dialects", Selected Proceedings of the 10th Hispanic Linguistics Symposium. Eds. Joyce Bruhn de Garavito y Elena Valenzuela. Somerville, Massachusetts: Cascadilla Proceedings Project. 276-287.

— 2008 b. "Speech rhythm variation in dialects of Spanish: Applying the Pairwise Variability Index and Variation Coefficients to Peruvian Spanish". Proceedings of Speech Prosody 008: Fourth Conference on Speech Prosody, Campinas, Brasil. 431-434.

Pamies Bertrán, Antonio y Mari Cruz Amorós Céspedes. 2005. "Pico tonal, acento y fronteras morfo-semánticas: Exprimento con hablantes granadinos". Estudios de Fonética Experimental XIV. 201-223.

— y Ana María Fernández Planas. 2006. "Sobre la percepción de la duración vocálica en español”. Actas del V Congreso Andaluz de Lingüística General. Homenaje a José Andrés de Molina. Ed. Juan de Dios Luque Durán. Granada. 501-512.

Pointon, Graham. 1978. A Contribution to the Study of Rhythm in Spanish. Edimburgo: University of Edinburgh.

—. 1980. "Is Spanish really syllable-timed?”. Journal of Phonetics 8. 293-304.

Prieto, Pilar. 2006. "Phonological phrasing in Spanish". Optimality-Theoretic Advances in Spanish Phonology. Eds. Sonia Colina y Fernando Martínez-Gil. Amsterdam y Filadelfia: John Benjamins. 39-60.

Ramus, Franck. 1999. Rythme des langues et acquisition du langage, École des Hautes Études en Sciences Sociales. En línea: http://www. ehess.fr/centres /lscp/ persons/ramus/ these/; 9/09/2009.

_- Marina Nespor y Jacques Mehler. 1999. "Correlates of linguistic rhythm in speech". Cognition 73. 265-292.

Rao, Rajiv. 2006. "On intonation's relationship with pragmatic meaning in Spanish". Selected Proceedings of the 8th Hispanic Linguistics Symposium. en Eds. Timothy Face y Carol A. Klee. Somerville, Massachusetts: Cascadilla Proceedings Project. 103-115.

—. 2007. "On the phonological phrasing patterns in the Spanish of Lima, Perú". Southwest Journal of Linguistics 26. 81-111.

- 2008. "Observations on the roles of prosody and syntax in the phonological phraing of Barcelona Spanish". The Linguistic Journal 3, 3. 85-131.

Roach, Peter. 1982 "On the distinction between 'stress-timed' and 'syllable-timed' languages". Linguistic Controversies: Essays in Linguistic Theory and Practice in Honour of F. R. Palmer. Ed. David Crystal. Londres: Edward Arnold Publishers Ltd. 73-79. 
Simón Casas, Francisco Javier. 2007. "Análisis de la entonación de los enunciados declarativos e interrogativos sin expansión en un hablante masculino de Jaca (Huesca)". III Congreso Internacional de Fonética Experimental. Eds. Manuel González González, Elisa Fernández Rei y Begoña González Rei. Santiago de Compostela: Xunta de Galicia. 561-567.

Toledo, Guillermo. 1987. "Patrones temporales en el español americano". Revista Argentina de Lingüística 3, 1. 55-68.

—. 1988 a. El ritmo en el español. Madrid: Gredos.

—. 1988 b. "Grouping and rhythm in Spanish discourse modes". The Study of Sounds 22, Ed. Onishi. Japón: The Phonetic Society of Japan. 177-186.

—. 1989. "Alternancia y ritmo en el español”. Estudios Filológicos 24. 19-30.

—. 1994. "Compresión rítmica en el español caribeño: habla espontánea”. Estudios de Fonética Experimental VI. 187-217.

1996. “Alternancia y ritmo en el español: habla espontánea”. Estudios Filológicos 31. $119-127$.

1997. "Prominencia melódica y temporal: El caso de la alternancia rítmica". Estudios de Fonética Experimental VIII. 153-183.

—. 1998. "Prominencia melódica y temporal: La colisión acentual en el español". Estudios de Fonética Experimental IX. 201-220.

—. 2008. "Frases fonológicas ( $\phi)$ ”. Ianua. Revista Philologica Romanica 8. 1-18.

—. Enviado. "Métricas rítmicas en un dialecto andaluz". Revista de Filología (Revista de Filología de la Universidad de La Laguna, Tenerife).

—. Mónica Trípodi, Jorge Gurlekian y Reina Yanagida. 2009. "Amper-Argentina: métricas rítmicas en dos corpus con diferencias socioeducativas". Comunicación en las V Jornadas Internacionales de Educación Lingüísticas: Lenguaje y Comunicación, Realidades y Desafios, Entre Ríos, Argentina.

Wenck, B. J. y Wioland, François. 1982. "Is French really syllable-timed?”. Journal of Phonetics 10. 203-216.

White, Laurence y Mattys, Sven.2007. "Rhythmic typology and variationin first and second languages”, en Pilar PRIETO, Joan Mascaró y María-José Solé (eds.), Segmental and Prosodic Issues in Romance Phonology, Current Issues in Linguistic Theory Series. Amsterdam y Filadelfia: John Benjamins. 237-257. 